\title{
Design, Synthesis, and Anti-inflammatory Activity of Novel Quinazolines
}

\author{
SAID A. EL-FEKY, MOHD. IMRAN and NAIRA NAYEEM \\ Department of Pharmaceutical Chemistry, Faculty of Pharmacy, Northern Border University, \\ Rafha 91911, P.O. Box 840, Kingdom of Saudi Arabia. \\ ${ }^{*}$ Corresponding author E-mail: drsaid.elfeky@yahoo.com \\ http://dx.doi.org/10.13005/ojc/330217
}

(Received: March 10, 2017; Accepted: March 30, 2017)

\begin{abstract}
Several new fluorinated quinazolinone derivatives were prepared and evaluated for in vitro anti-inflammatory activity. The molecular modelling study was performed for compounds 4, 8, 9, 10 and 13. The tested compounds showed strong interactions at the COX-2 binding sites. Compounds $8,13,9$, and 10 containing triazole, thiadiazole, and oxadiazole rings showed the highest in vitro anti-inflammatory activity and the best binding into the COX-2 binding site.
\end{abstract}

Keywords: Design, Fluorinated quinazolinones, Molecular modelling study, in vitro anti-inflammatory activity.

\section{INTRODUCTION}

Heterocyclic compounds form the basis of many pharmaceuticals, agrochemicals, and veterinary products. Among a wide variety of nitrogen heterocyclic moieties, quinazolinone possess a diverse biological activity profile. The pharmacodynamics versatility of quinazolin-4one moiety has been documented not only in its synthetic derivatives, but also in several naturally occurring alkaloids isolated from animals, plants, and microorganisms, for example, tryptahthin and rutaecarpine. Also proquazone and fluproquazone are non-acidic anti-inflammatory drugs ${ }^{1,2}$. Celecoxib is a selective COX-2 inhibitory anti-inflammatory drug and has been reported to possess some side effects. Therefore many efforts were performed to develop lead compounds possessing greater efficacy and less side effects than celecoxib ${ }^{3}$. Fluorine atom plays an important role in medicinal chemistry ${ }^{4,5}$. The presence of fluorine atom enhances the pharmacokinetic, the physicochemical properties and the binding to the targeted protein. ${ }^{6}$. Previously, we have synthesized a number of quinoline derivatives bearing a fluorine atom such as compound V (Fig. 1) which exhibited high anti-inflammatory activity in comparison to the celecoxib ${ }^{7}$. On the basis of bioisosterism concept, it was a dream of interest to synthesize compounds 
1-17 in the hope that one of them may become a lead compound having potent anti-inflammatory activity $^{8,9}$.

\section{MATERIAL AND METHODS}

\section{Chemistry}

Melting points were obtained by open capillary method using Barnstead 9100 electrothermal melting apparatus. IR spectra $(\mathrm{KBr})$ were generated using Perkin-Elmer spectrometer $\left(\mathrm{I}_{\mathrm{cm}} \mathrm{cm}^{-1}\right.$ ). The ${ }^{1} \mathrm{H}$ NMR spectra were obtained from Varian Gemini300 spectrophotometer. The ${ }^{13} \mathrm{C}-\mathrm{NMR}$ spectra were generated on Brucker $500 \mathrm{MHz}$ spectrophotometer. The mass spectra were obtained with the help of a Perkin-Elmer, Clarus 600 TGC / MS, spectrometer.

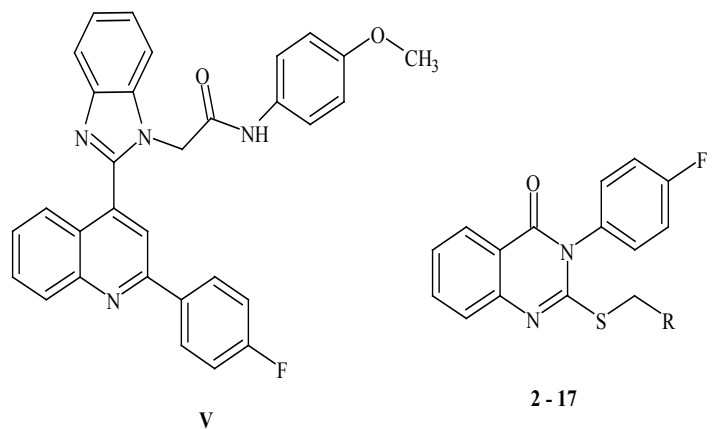

Flg. 1: Bioisosteric concept
2-Mercapto-3-(4-fluorophenyl)-3H-quinazolin-4one (1)

Anthranilic acid ( $1 \mathrm{mmol}$ ) and 4-fluorophenylisothiocyanate $(1.5 \mathrm{mmol})$ in $50 \mathrm{ml}$ ethanol was refluxed for 4 hours. The reaction mixture was cooled at room temperature and the obtained solid was dried and recrystallized from ethanol to give compound 1 in $95 \%$ yield. m.p. > $300^{\circ} \mathrm{C}$; IR: 1695 $(\mathrm{C}=\mathrm{O}), 2650$ (-SH); ${ }^{1} \mathrm{H}-\mathrm{NMR}$ (DMSO-D,$\left.\delta \mathrm{ppm}\right)$ : 6.95-8.20 (m, 4H, Ar-H of 4-Fluorophenyl), 8.50-8.55 (m, $4 \mathrm{H}$, Ar- $\mathrm{H}$ of quinazoline), $12.96(\mathrm{~s}, 1 \mathrm{H})$; Mass (m/z): $272\left[\mathrm{M}^{+}\right]$; Elemental Analysis $\left(\mathrm{C}_{14} \mathrm{H}_{9} \mathrm{FN}_{2} \mathrm{OS}\right)$ : Calcd. C, 61.75; H, 3.33; N, 10.29; Found: C, 61.52; $\mathrm{H}, 3.47 ; \mathrm{N}, 10.41$.

Ethyl-2-[3-(4-fluorophenyl)-3,4-dihydro-4oxoquinazolin-2-yl)thio]acetate (2)

Compound 1 (1 $\mathrm{mmol}$ ) and ethyl 2-bromoacetate $(1.5 \mathrm{mmol})$ in $15 \mathrm{ml}$ of acetone containing $\mathrm{K}_{2} \mathrm{CO}_{3}(1.5 \mathrm{mmol})$ was stirred for 10 hours at room temperature. The precipitated solid was filtered, and crystallized from ethanol to give compound 2 in $80 \%$ yield. m.p. $130-132{ }^{\circ} \mathrm{C}$; IR: 1692 and $1735(\mathrm{C}=\mathrm{O})$; ${ }^{1} \mathrm{H}-\mathrm{NMR}: 1.56(\mathrm{t}, 3 \mathrm{H})$, 4.02 (s, 2H), 4.30-4.70 (q, 2H), 6.95-8.2 (m, 4H, Ar-H of 4-fluorophenyl), 8.5-8.55 (m, 4H, Ar-H of quinazoline); ${ }^{13} \mathrm{C}-\mathrm{NMR}: 14.11,30.81,60.62,115.77$, $115.69,119.41,120.91,126.66,126.77,127.33$, $128.33,130.11,133.41,146.99,159.35,160.61$ 162.91, 169.41; Mass (m/z): $358\left(\mathrm{M}^{+}\right)$; Elemental

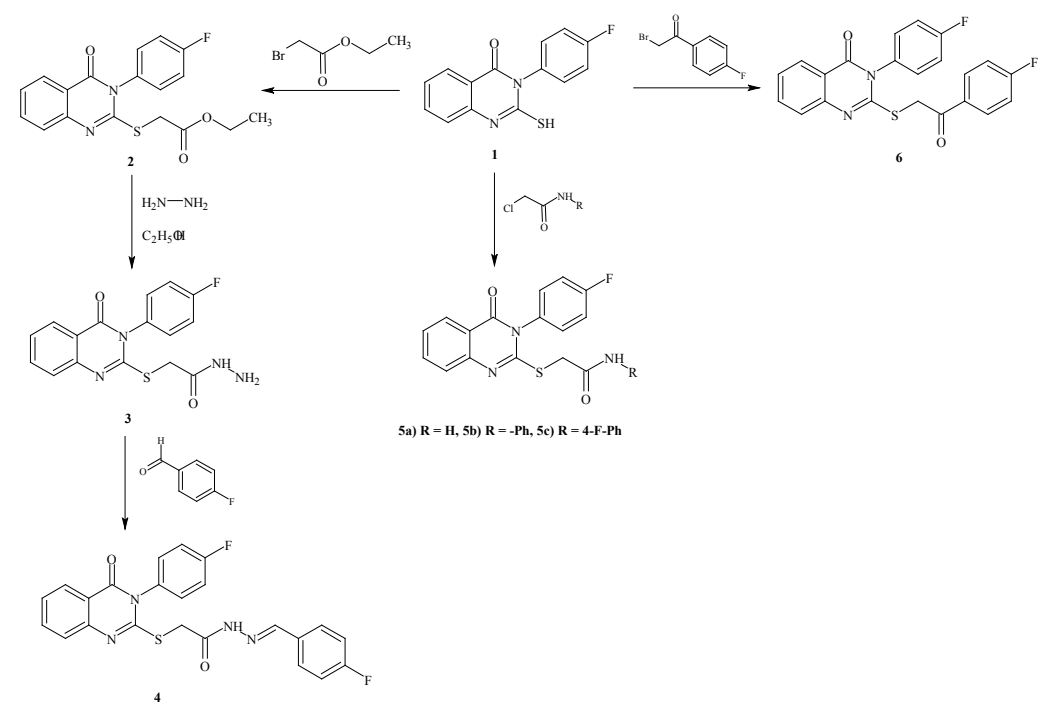

Scheme 1 
Analysis $\left(\mathrm{C}_{18} \mathrm{H}_{15} \mathrm{FN}_{2} \mathrm{O}_{3} \mathrm{~S}\right)$ : Calcd. C, 60.33; $\mathrm{H}, 4.22$; N, 7.82; Found: C, 60.21; H, 4.11; N, 7.86.

\section{2 - [ 3 - ( 4 - flu oropheny ) - 3, 4 - dihy d ro - 4 - oxoquinazolin-2-yl)thio]acetohydrazide (3)}

Hydrazine hydrate $(2 \mathrm{mmol})$ was added to compound $2(1 \mathrm{mmol})$ in $10 \mathrm{ml}$ absolute ethanol and the reaction mixture was stirred for 12 hours. The solid obtained was filtered and recrystallized from ethanol to give compound 3 in $85 \%$ yield. m.p. 170-172 ${ }^{\circ} \mathrm{C}$; IR: 1688 and 1660 (C=O), 3311, 3290, 3245 (N-H); ${ }^{1} \mathrm{H}-\mathrm{NMR}: 4.22$ (s, 2H), 4.31 (s, 2H), 6.95-8.1 (m, 4H, Ar-H of 4-fluorophenyl), 8.50-8.56 ( $\mathrm{m}, 4 \mathrm{H}, \mathrm{Ar}-\mathrm{H}$ of quinazoline), $9.18(\mathrm{~s}, 1 \mathrm{H}) ;{ }^{13} \mathrm{C}-\mathrm{NMR}$ : $30.44,115.71,115.72,120.81,126.61,126.73$, 127.32, 128.31, 130.11, 133.42, 146.22, 146.91, 159.33, 160.61, 162.96, 170.31; Mass (m/z): 344 $\left(\mathrm{M}^{+}\right)$; ; Elemental Analysis $\left(\mathrm{C}_{16} \mathrm{H}_{13} \mathrm{FN}_{4} \mathrm{O}_{2} \mathrm{~S}\right)$ : Calcd. $\mathrm{C}$,

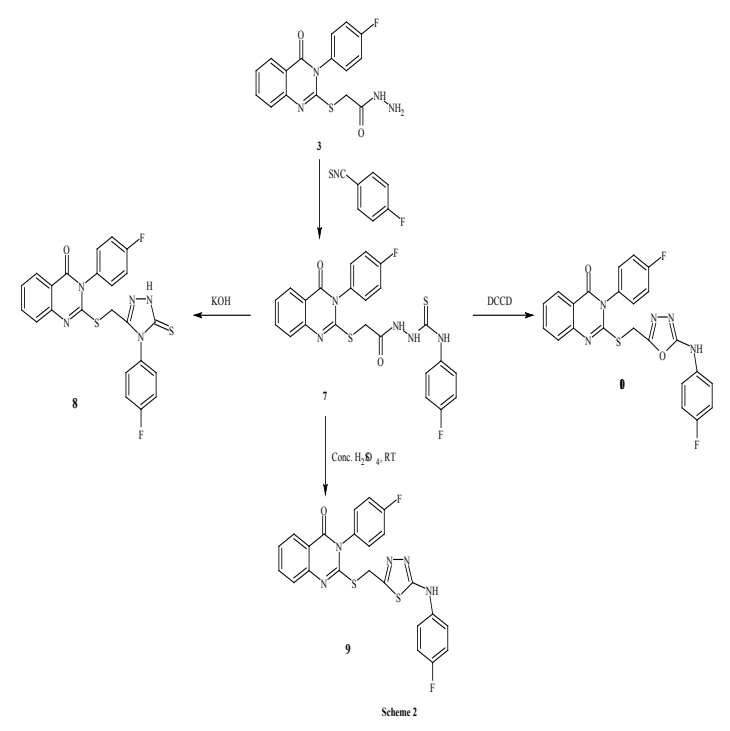

Scheme 2

Table 1: Values for in vitro COX-1 / COX-2 enzyme inhibition by the designed compounds

\begin{tabular}{llll}
\hline Compound No. & COX-1 & COX-2 & SI \\
\hline $\mathbf{4}$ & $>100$ & 0.33 & $>303.0$ \\
$\mathbf{8}$ & $>100$ & 0.83 & $>125.0$ \\
$\mathbf{9}$ & $>100$ & 0.42 & $>250$ \\
$\mathbf{1 0}$ & $>100$ & 0.73 & $>142.9$ \\
$\mathbf{1 3}$ & $>100$ & 0.31 & $>333.3$ \\
\hline
\end{tabular}

55.81; H, 3.81; N, 16.27; Found: C, 55.66; H, 3.86; N, 16.31 .

(E)-N-(4-fluorobenzylidene)-2-[3-(4fluorophenyl)-3,4-dihydro-4-oxoquinazolin-2-yl) thio] acetohydrazide (4)

Compound $3(1.5 \mathrm{mmol})$ and p-fluorobenzaldehyde $(1.5 \mathrm{mmol})$ in $15 \mathrm{ml}$ of absolute ethanol was stirred for 1 hour. The obtained solid was filtered and crystallized from ethanol to give compound 4 in $81 \%$ yield. m.p. $182-184^{\circ} \mathrm{C}$; IR: 1687 and $1655(\mathrm{C}=\mathrm{O}), 3315(-\mathrm{NH}-),{ }^{1} \mathrm{H}-\mathrm{NMR}: 4.18(\mathrm{~s}, 2 \mathrm{H})$, 6.96-8.10 (m, 4H, Ar-H of 4-fluorophenyl), 8.50-8.55 ( $\mathrm{m}, 4 \mathrm{H}, \mathrm{Ar}-\mathrm{H}$ of quinazoline), $10.95(\mathrm{~s}, 0.5 \mathrm{H}), 11.05$ (s, 0.5H); ${ }^{13} \mathrm{C}-\mathrm{NMR}: 30.77,115.61,115.61,116.71$ (2C), 120.81, 126.63, 126.71, 127.33, 128.33, $129.38,130.16,130.16,130.81,138.11,138.82$, 144.11, 146.11, 148.11, 160.61, 162.93, 165.22, 171.11; Mass (m/z): $450\left(\mathrm{M}^{+}\right)$; Elemental Analysis $\left(\mathrm{C}_{23} \mathrm{H}_{16} \mathrm{~F}_{2} \mathrm{~N}_{4} \mathrm{O}_{2} \mathrm{~S}\right)$ : Calcd. C, 61.33; $\mathrm{H}, 3.58 ; \mathrm{N}, 12.44$; Found: C, 61.21; H, 3.55; N, 12.51.

\section{Synthesis of compounds 5a-c}

To compound 1 (2 mmol) in $15 \mathrm{ml}$ acetone, the respective 2-chloro- $\mathrm{N}$-(substitutedphenyl) anilide $(2.1 \mathrm{mmol})$ and anhydrous $\mathrm{K}_{2} \mathrm{CO}_{3}(3 \mathrm{mmol})$ was added and refluxed with stirring for 1 hour. The precipitated solid was filtered, washed with water, and recrystallized from ethanol.

2-[3-(4-fluorophenyl)-3, 4-dihydro-4oxoquinazolin-2-yl)thio]acetamide (5a) Yield $85 \%$; m.p. $177-179{ }^{\circ} \mathrm{C}$; IR: 1682 and $1651(\mathrm{C}=\mathrm{O}), 3180(\mathrm{~N}-\mathrm{H}) ;{ }^{1} \mathrm{H}-\mathrm{NMR}: 4.19(\mathrm{~s}, 2 \mathrm{H})$, 6.91-8.10 (m, 4H, Ar-H of 4-fluorophenyl), 8.50-8.55 ( $\mathrm{m}, 4 \mathrm{H}, \mathrm{Ar}-\mathrm{H}$ of quinazoline), $8.54(\mathrm{~s}, 1 \mathrm{H}) ;{ }^{13} \mathrm{C}-\mathrm{NMR}$ : $30.92,115.71,115.72,120.81,126.62,126.63$, $126.71,127.33,128.33,130.11,133.41,146.91$, 159.31, 160.63, 162.92, 170.81; Mass (m/z): 329 $\left(\mathrm{M}^{+}\right)$; Elemental Analysis $\left(\mathrm{C}_{16} \mathrm{H}_{12} \mathrm{FN}_{3} \mathrm{O}_{2} \mathrm{~S}\right)$ : Calcd. C, 58.35; H, 3.67; N, 12.76; Found: C, 58.22; H, 3.55; $\mathrm{N}, 12.55$.

2-[3-(4-fluoropheny I)-3, 4-dihydro-4oxoquinazolin-2-yl)thio]- $\mathrm{N}$-phenylacetamide (5b)

Yield 95\%; m.p. 162-164 ${ }^{\circ} \mathrm{C}$; IR: 1683 and $1655(\mathrm{C}=\mathrm{O}), 3183(\mathrm{~N}-\mathrm{H})$; ${ }^{1} \mathrm{H}-\mathrm{NMR}: 4.22$ (s, 2H), 6.92-8.30 (m, 8H, Ar-H), 8.50-8.55 (m, 4H, Ar-H of quinazoline), 8.57 (s, 1H); ${ }^{13} \mathrm{C}-\mathrm{NMR}: 28.33,115.71$, 
$115.72,121.61,121.62,126.61,126.72,127.31$, $128.11,128.81,128.33,128.91,130.32$ (2C), 132.44, 133.41, 139.51, 146.92, 159.31, 160.61, 162.91, 168.22; Mass (m/z): $405\left(\mathrm{M}^{+}\right)$; Elemental Analysis $\left(\mathrm{C}_{22} \mathrm{H}_{16} \mathrm{FN}_{3} \mathrm{O}_{2} \mathrm{~S}\right)$ : Calcd. C, 65.17; $\mathrm{H}, 3.98 ; \mathrm{N}, 10.36$; Found: C, 65.22; H, 3.65; N, 10.22 .

2 - [ 3 - ( 4 - fluorophen y I) - 3, 4 - dih y d ro - 4 oxoquinazolin-2-yl)thio]-N-(4-fluorophenyl) acetamide (5c)

Yield 85\%; m.p. 152-154 ${ }^{\circ} \mathrm{C}$; IR: 1682 and $1654(\mathrm{C}=\mathrm{O}), 3182(\mathrm{~N}-\mathrm{H})$; ${ }^{1} \mathrm{H}-\mathrm{NMR}: 4.22$ (s, 2H), 6.93-8.33 (m, 8H, Ar-H), 8.50-8.56 (m, 4H, Ar-H of quinazoline), 8.58 (s, $1 \mathrm{H}) ;{ }^{13} \mathrm{C}-\mathrm{NMR}: 29.22,81.21$, $110,11,115.71,115.72,120.81,125.71,126.61$, 126.71, 127.31, 128.33, 130.11 (2C), 131.33, 133.41, 137.51, 137.51, 146.91, 159.31, 160.61, 162.91, 167.33; Mass (m/z): $423\left(\mathrm{M}^{+}\right)$; Elemental Analysis

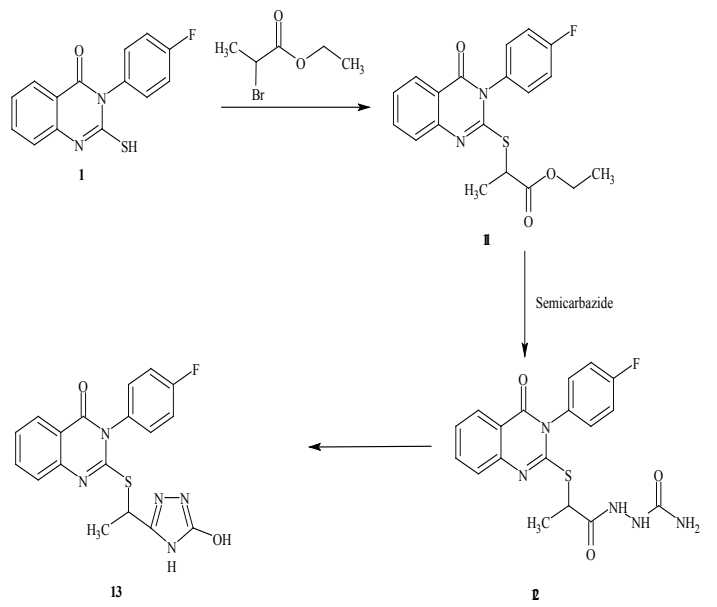

Scheme 3

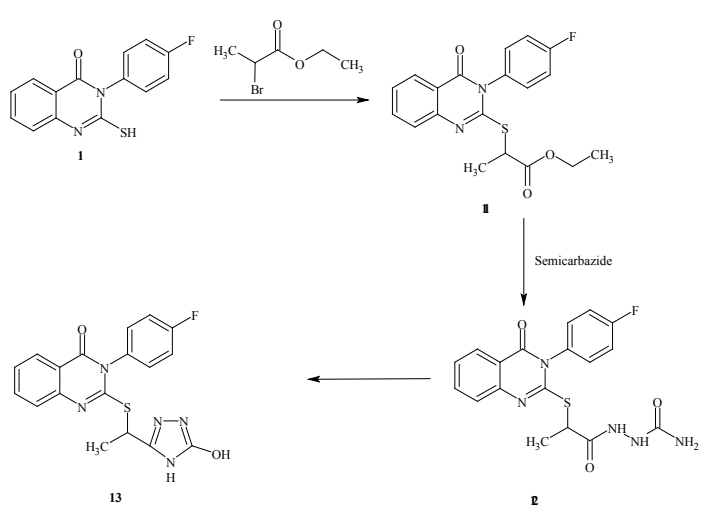

Scheme 3 : Synthesis and cycli zation of $4(3 \mathrm{H})-$ quinazoline bearing 1,2 , 4- triazole ring
$\left(\mathrm{C}_{22} \mathrm{H}_{15} \mathrm{~F}_{2} \mathrm{~N}_{3} \mathrm{O}\right.$ S $)$ : Calcd. C, 62.40; H, 3.57; N, 9.92; Found: C, 62.33; H, 3.81; N, 9.88.

2-(4-fluorophenacyl)thio]-3-(4-fluorophenyl)-3,4dihydro-4-oxoquinazolin (6)

Compound 1 (1 mmol) in $20 \mathrm{ml}$ acetone, 4-fluorophenacyl bromide $(1.1 \mathrm{mmol})$ and $\mathrm{K}_{2} \mathrm{CO}_{3}(2$ $\mathrm{mmol}$ ) was heated under reflux for 5 hours. Ice cold water was added to the reaction mixture and the solid obtained was filtered and recrystallized from ethanol to give compound 6 in $71 \%$ yield. m.p. $157-159{ }^{\circ} \mathrm{C}$; IR: 1682 and $1654(\mathrm{C}=\mathrm{O})$; ${ }^{1} \mathrm{H}-\mathrm{NMR}: 3.25(\mathrm{~s}, 1 \mathrm{H})$, 4.22 (s, 2H), 6.96-8.3 (m, 8H, Ar-H), 8.50-8.55 (m, $4 \mathrm{H}, \mathrm{Ar}-\mathrm{H}$ of quinazoline); ${ }^{13} \mathrm{C}-\mathrm{NMR}$ : $35.81,115.41$, 115.42, 115.71, 115.72, 120.81, 126.61, 126.71, $127.31,128.31,130.11$ (2C), 131.0, 131.11 (2C), 133.41, 146.91, 159.31, 160.61, 162.91, 167.33, 194.11; Mass (m/z): $408\left(\mathrm{M}^{+}\right)$; Elemental Analysis $\left(\mathrm{C}_{22} \mathrm{H}_{14} \mathrm{~F}_{2} \mathrm{~N}_{2} \mathrm{O}\right.$ S $)$ : Calcd. C, 64.70; H, 3.46; N, 6.86; Found: C, 64.66; H, 3.55; N, 7.11.

2-[2-[3-(4-fluorophenyl)-3,4-dihydro4-oxoquinazolin-2-yl]thio] acetyl-N-(4fluorophenylhydrazine)-1-carbothioamide (7)

Compound $3(2 \mathrm{mmol})$ in $20 \mathrm{ml}$ absolute ethanol was refluxed with 4-fluorophenylisothiocyanate $(2.1 \mathrm{mmol})$ for 1 hour. The obtained solid was filtered and recrystallized from ethanol to give compound 7 in $83 \%$ yield. m.p. $171-173^{\circ} \mathrm{C}$; IR: 3384,3187 (2 $\mathrm{N}-\mathrm{H}), 1676,1650(\mathrm{C}=\mathrm{O})$ and $1350(\mathrm{C}=\mathrm{S}) ;{ }^{1} \mathrm{H}-\mathrm{NMR}$ : $4.01(\mathrm{~s}, 2 \mathrm{H}), 6.94-8.20(\mathrm{~m}, 8 \mathrm{H}, \mathrm{Ar}-\mathrm{H}), 8.51-8.56$ (m, $4 \mathrm{H}, \mathrm{Ar}-\mathrm{H}$ of quinazoline), $10.33(\mathrm{~s}, 1 \mathrm{H}), 9.24(\mathrm{~s}$, 1H), 8.61 (s, 1H); ${ }^{13} \mathrm{C}-N M R: 31.11,115.71,115.72$, 115.81, 115.82, 120.81, 126.61, 126.71, 127.32, 128.31, 128.32, 130.11, 131.11 (2C), 131.22, 133.41, 146.91, 159.33, 160.62, 162.91, 163.33, 170.33, 181.11; Mass (m/z): $497\left(\mathrm{M}^{+}\right)$; Elemental Analysis $\left(\mathrm{C}_{23} \mathrm{H}_{17} \mathrm{~F}_{2} \mathrm{~N}_{5} \mathrm{O}_{2} \mathrm{~S}_{2}\right)$ : Calcd. C, 55.52; $\mathrm{H}, 3.44 ; \mathrm{N}, 14.08$; Found: C, 55.66; $\mathrm{H}, 3.35 ; \mathrm{N}, 14.11$.

2-([(4-fluorophenyl-5-mercapto-4H-1,2,4-triazol-3yl)methyl]thio)-3-(4-fluorophenyl)-3,4-dihydro-4oxoquinazolin (8)

Compound 7 (2 mmol) was refluxed with aqueous $\mathrm{KOH}$ ( $3 \mathrm{mmol}$ ) for 2 hours. The clear solution was neutralized with $10 \% \mathrm{HCl}$ and the obtained solid was filtered, washed with water and recrystallized from ethanol to give compound 8 in $81 \%$ yield. m.p. 221-223 ${ }^{\circ} \mathrm{C}$; IR: 1687 (C=O); ${ }^{1} \mathrm{H}-\mathrm{NMR}: 4.47$ (s, 2H), 6.93-8.31 (m, 8H, Ar-H), 8.51-8.53 (m, 4H, Ar-H of 
Table 2: Molecular modeling data for compounds 4, 8, 9, 10, 13 and Celecoxib during docking in COX-2 (PDB ID: 1CX2) active site

\begin{tabular}{|c|c|c|c|c|c|c|}
\hline \multirow[t]{2}{*}{ Compound } & \multirow[b]{2}{*}{$\begin{array}{l}\text { Affinity } \\
\text { Kcal/mol }\end{array}$} & \multirow[b]{2}{*}{$\begin{array}{l}\text { Distance (in } \mathrm{A}^{\circ} \text { ) } \\
\text { from } \\
\text { main residue }\end{array}$} & \multirow[b]{2}{*}{$\begin{array}{l}\text { Functional } \\
\text { group }\end{array}$} & \multicolumn{2}{|l|}{ coX-2 } & \\
\hline & & & & Interaction & 2d caption & \\
\hline \multirow[t]{3}{*}{4} & 0.9 & 3.01 & Glu524 & $-S-$ & H-donor & Fig. 2 \\
\hline & -5.3 & 3.13 & Glu524 & $-S-$ & H-donor & \\
\hline & -1.0 & 3.88 & Arg120 & -Ph-ring & pi-cation & \\
\hline \multirow[t]{5}{*}{8} & -0.6 & 4.03 & Val349 & -S- & H-donor & Fig. 3 \\
\hline & -0.1 & 3.40 & & $-S-$ & H-donor & \\
\hline & -1.1 & 3.31 & Leu352 & $=\mathrm{N}-$ & $\mathrm{H}$-acceptor & \\
\hline & -2.2 & 2.94 & Ser353 & $=\mathrm{S}$ & & \\
\hline & -1.3 & 3.60 & $\begin{array}{l}\text { Arg513 } \\
\text { Arg513 }\end{array}$ & $=S$ & $\begin{array}{l}\text { H-acceptor } \\
\text { H-acceptor }\end{array}$ & \\
\hline \multirow[t]{2}{*}{9} & -1.4 & 3.70 & Lys83 & -S- & H-acceptor & Fig. 4 \\
\hline & -0.6 & 4.36 & Arg513 & -Ph-ring & pi-cation & \\
\hline \multirow[t]{3}{*}{10} & -5.1 & 2.82 & Glu524 & $-\mathrm{NH}-$ & H-donor & Fig. 5 \\
\hline & -11.3 & 3.06 & Lys83 & $=\mathrm{N}-$ & H-acceptor & \\
\hline & -0.7 & 4.08 & Arg513 & -Ph-ring & pi-cation & \\
\hline \multirow[t]{2}{*}{13} & -1.2 & 3.13 & Arg120 & $=\mathrm{N}-$ & $\mathrm{H}$-acceptor & Fig. 6 \\
\hline & -1.8 & 3.14 & Arg120 & $=\mathrm{N}-$ & H-acceptor & \\
\hline \multirow[t]{2}{*}{ Celecoxib } & -6.5 & 2.91 & Arg513 & $-\mathrm{SO}_{2}$ & H-acceptor & Fig. 7 \\
\hline & & 2.51 & Arg120 & $-\mathrm{SO}_{2}$ & H-acceptor & \\
\hline
\end{tabular}

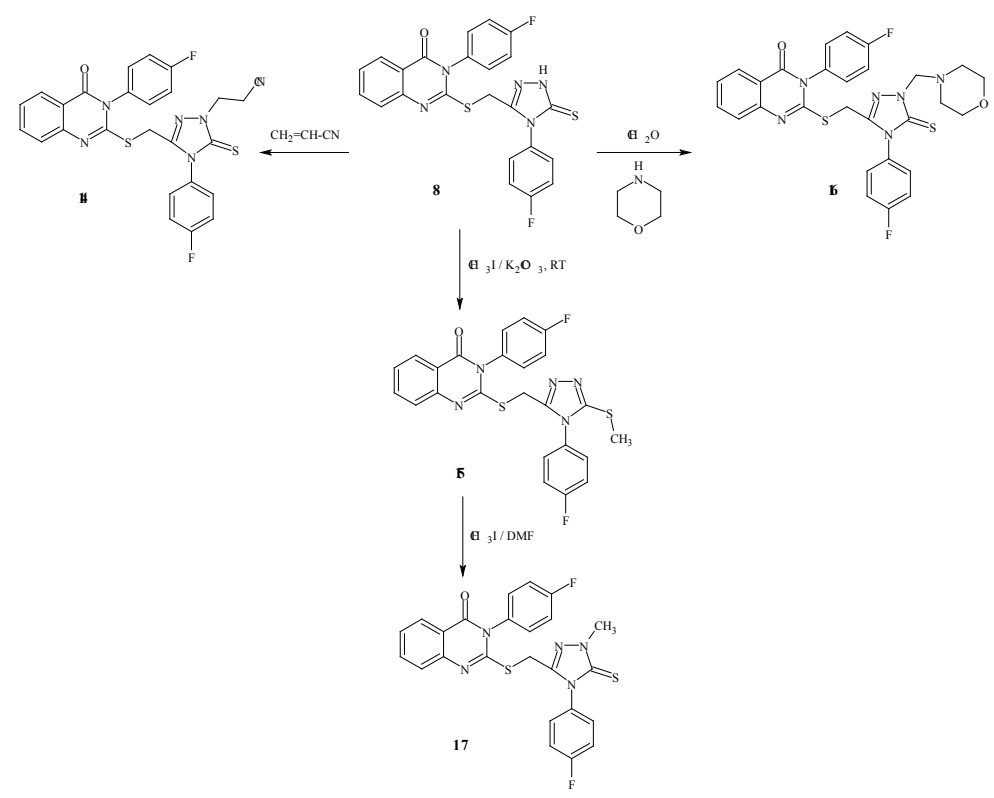

Scheme 4 
quinazoline), 13.89 (s, 1H); ${ }^{13} \mathrm{C}-\mathrm{NMR}: 29.11,115.71$ (2C), 115.81 (2C), 120.81, 126.61, 127.33, 128.31, $129.51,130.11$ (2C), 133.41, 135.11, 135.12, $146.91,157.11,159.33,160.63,162.92,163.31$, 166.71; Mass (m/z): $479\left(\mathrm{M}^{+}\right)$; Elemental Analysis $\left(\mathrm{C}_{23} \mathrm{H}_{15} \mathrm{~F}_{2} \mathrm{~N}_{5} \mathrm{OS}_{2}\right)$ : Calcd. C, 57.61; $\mathrm{H}, 3.15 ; \mathrm{N}, 14.61$; Found: C, 57.55; H, 3.16; N, 14.21.

2-([(5-(4-fluorophenyl)amino)1,3,4-thiadiazole-2yl)methyl]thio)-3-(4-fluorophenyl)-3,4-dihydro-4oxoquinazolin (9)

Compound $7(2 \mathrm{mmol})$ and concentrated $\mathrm{H}_{2} \mathrm{SO}_{4}(5 \mathrm{ml})$ was stirred at room temperature for 12 hours. The reaction mixture was neutralized with $\mathrm{KHCO}_{3}$ and the obtained solid was crystallized to give compound $974 \%$ yield. m.p. $228-230{ }^{\circ} \mathrm{C}$; IR: $3182(\mathrm{~N}-\mathrm{H})$ and $1683(\mathrm{C}=\mathrm{O})$; ${ }^{1} \mathrm{H}-\mathrm{NMR}: 4.67(\mathrm{~s}, 2 \mathrm{H})$, 6.92-8.23 (m, 8H, Ar-H), 8.52-8.56 (m, 4H, Ar-H of quinazoline), 9.82 (s, $1 \mathrm{H}, \mathrm{NH}) ;{ }^{13} \mathrm{C}-\mathrm{NMR}: 24.41$, $115.71,115.72,116.31,116.33,120.62,120.82$, $126.62,126.72,127.34,128.33,128.36,130.11$, 133.41, 138.11, 146.91, 152.72, 157.31, 159.32, 159.33, 160.61, 162.92, 168.01; Mass (m/z): 479 $\left(\mathrm{M}^{+}\right)$; Elemental Analysis $\left(\mathrm{C}_{23} \mathrm{H}_{15} \mathrm{~F}_{2} \mathrm{~N}_{5} \mathrm{OS}\right)_{2}$ : Calcd. C, 57.61; H, 3.15; N, 14.61; Found: C, 57.55; H, 3.33; N, 14.75 .

2-([(5-(4-fluorophenyl)amino)-1,3,4-oxadiazol-2yl)methyl]thio-3-(4-fluorophenyl)-3,4-dihydro-4oxoquinazolin (10)

To compound 7 (1 mmol) in toluene (20 $\mathrm{ml})$, DCCD (1.5 mmol) was added and the reaction mixture was refluxed for 6 hours. On cooling the obtained solid was filtered and recrystallized from ethanol to give compound 10 in $63 \%$ yield. m.p.
271-273 ${ }^{\circ} \mathrm{C}$; IR: $3182(\mathrm{~N}-\mathrm{H})$ and $1686(\mathrm{C}=\mathrm{O}) ;{ }^{1} \mathrm{H}-$ NMR: 4.62 (s, 2H), 4.68 (s, 1H), 6.91-8.28 (m, 8H, Ar-H), 8.52-8.56 (m, 4H, Ar-H of quinazoline), 9.88 (s, 1H); ${ }^{13} \mathrm{C}-\mathrm{NMR}: 24.41,115.71,115.72,116.32$, 116.33, 116.34, 120.61, 120.62, 120.64, 120.82, $126.71,127.33,128.34,130.11,130.13,134.51$, 146.92, 157.33, 159.32, 160.61, 162.92, 163.24, 169.33; Mass (m/z): $463\left(\mathrm{M}^{+}\right)$; Elemental Analysis $\left(\mathrm{C}_{23} \mathrm{H}_{15} \mathrm{~F}_{2} \mathrm{~N}_{5} \mathrm{O}_{2} \mathrm{~S}\right)$ : Calcd. C, 69.61; H, 3.26; N, 15.11; Found: C, 69.55; H, 3.11; N, 15.22 .

Ethyl 2-(3-(4-fluorophenyl)-4-oxo-3,4dihydroquinazolin-2-yl) thio) propanoate (11)

Compound 1 (1 mmol) was dissolved in 15 $\mathrm{ml}$ of absolute ethanol and $\mathrm{KOH}(1.5 \mathrm{mmol})$. Ethyl ethyl-2-bromopropanoate $(1.5 \mathrm{mmol})$ was added dropwise to the reaction mixture and refluxed for 6 hours. The reaction mixture was poured on ice cold water and the precipitated solid was filtered, and recrystallized from ethanol to give compound 11 in 75\% yield. m.p. $190-192{ }^{\circ} \mathrm{C}$; IR: 1733 (C=O, ester), $1687\left(\mathrm{C}=\mathrm{O}\right.$, quinazolinone); ${ }^{1} \mathrm{H}-\mathrm{NMR}: 0.82(\mathrm{t}, 3 \mathrm{H})$, 1.19 (d, 3H), 2.73 (q, 1H), 3.32 (q, 2H), 7.20-8.20 (m, 8H, Ar-H); ${ }^{13} \mathrm{C}-\mathrm{NMR}: 14.11,18.52,37.11,60.92$, $115.72,120.81,126.61,126.72,128.72,128.33$, $130.11,130.12,133.41,146.92,155.74,159.32$, 160.62, 162.92, 170.82; Mass (m/z): $372\left(\mathrm{M}^{+}\right)$; Elemental Analysis $\left(\mathrm{C}_{19} \mathrm{H}_{17} \mathrm{FN}_{2} \mathrm{O}_{3} \mathrm{~S}\right)$ : Calcd. C, 61.28; H, 4.60; N, 7.52; Found: C, 61.33; H, 4.77; N, 8.11.

2 - ( 3 - ( 4 - fluoropheny I) - 4 - oxo-3, 4 dihydroquinazolin-2-yl)thio)propanoyl semicarbazide (12)

Compound $30.11,130.16,133.44,146.91$, $157.41,159.33,160.62,162.93,174.82 ;$ Mass (m/z):

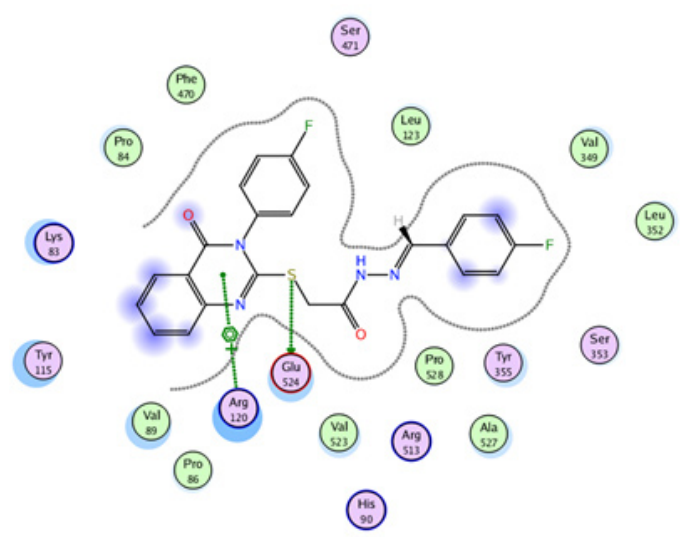

Fig. 2

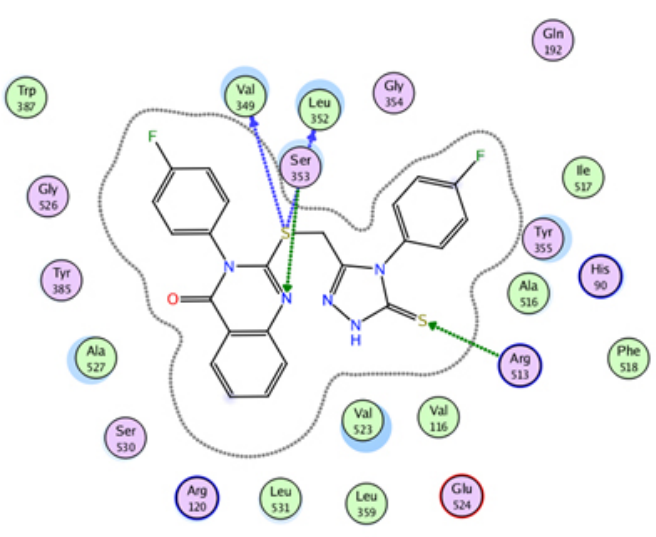

Fig. 3 
$401\left(\mathrm{M}^{+}\right)$; Elemental Analysis $\left(\mathrm{C}_{18} \mathrm{H}_{16} \mathrm{FN}_{5} \mathrm{O}_{3} \mathrm{~S}\right)$ : Calcd. C, 53.86; H, 4.02; N, 17.45; Found: C, 53.77; H, 4.11; N, 17.66 .

3 - [ 1 - ( 3 - ( 4 - f l u o rophe n y I) - 4 - ox o- 3, 4 dihydroquinazolin-2-yl-thio)ethyl]-4H-1,2,4triazol-5-ol (13)

Compound $12(1 \mathrm{mmol})$ and $20 \%$ aqueous $\mathrm{KOH}(25 \mathrm{ml})$ was refluxed for 12 hours. The mixture was cooled, poured into ice cold water, stirred and neutralized by gradual addition of (1:1) hydrochloric acid. The precipitate was filtered, washed with $\mathrm{H}_{2} \mathrm{O}$ and recrystallized from ethanol to give compound 13 in $90 \%$ yield. m.p. $>300{ }^{\circ} \mathrm{C}$; IR: $3444(\mathrm{O}-\mathrm{H})$, $3377(\mathrm{~N}-\mathrm{H}), 1685(\mathrm{C}=\mathrm{O}), 1639(\mathrm{C}=\mathrm{N}) ;{ }^{1} \mathrm{H}-\mathrm{NMR}$ : $1.58(\mathrm{~d}, 3 \mathrm{H}), 2.83$ (q, 1H), $5.1(\mathrm{~s}, 1 \mathrm{H}), 7.41-8.32$ (m, 8H, Ar-H), 9.81 (s, $1 \mathrm{H}) ;{ }^{13} \mathrm{C}-N M R: 17.91,33.81$, $115.72,115.75,120.81,126.61,127.32,128.33$, $128.73,131.11,131.12,133.44,148.92,152.11$, 159.33, 159.34, 160.61, 162.93; Mass (m/z): 383 $\left(\mathrm{M}^{+}\right)$; Elemental Analysis $\left(\mathrm{C}_{18} \mathrm{H}_{14} \mathrm{FN}_{5} \mathrm{O}_{2} \mathrm{~S}\right)$ : Calcd. C, 56.39; H, 3.68; N, 18.27; Found: C, 56.29; H, 3.55; $\mathrm{N}, 18.11$.

2-(1- $\beta$-cyanoethyl-4-(4-fluorophenyl)-5-thioxo- $\Delta$ s-triazolin-3-ylmethyl)-3-(4-fluorophenyl) -3,4dihydro-4-oxo-quinazolin (14)

The solution of compound $8(1 \mathrm{mmol})$ in water $(5 \mathrm{ml})$ and pyridine $(20 \mathrm{ml})$ was treated with acrylonitrile $(1 \mathrm{ml})$. The mixture was refluxed for 4 hours and the volume was reduced to half. The solid was filtered, washed with water and recrystallized from ethanol to give compound 14 in 50\% yield. m.p. 172-174 ${ }^{\circ} \mathrm{C}$; IR: 1682 (C=O), 2230 (CN); ${ }^{1} \mathrm{H}-\mathrm{NMR}$ : $1.16(\mathrm{t}, 2 \mathrm{H}), 3.98(\mathrm{t}, 2 \mathrm{H}), 4.20(\mathrm{~s}, 2 \mathrm{H}), 6.92-8.23(\mathrm{~m}$, $8 \mathrm{H}, \mathrm{Ar}-\mathrm{H}), 8.52-8.56(\mathrm{~m}, 4 \mathrm{H}, \mathrm{Ar}-\mathrm{H}$ of quinazoline); ${ }^{13} \mathrm{C}-N M R: 29.91,115.71,115.81,120.81,125.62$, $125.71,126.61,126.71,127.33,127.34,127.38$, $128.55,128.56,130.11,130.13,130.14,133.42$, 135.01, 135.03, 146.91, 157.81, 159.22, 160.61, 162.9, 167.61, 190.33; Mass (m/z): $532\left(\mathrm{M}^{+}\right)$; Elemental Analysis $\left(\mathrm{C}_{26} \mathrm{H}_{18} \mathrm{~F}_{2} \mathrm{~N}_{6} \mathrm{OS}\right)$ : Calcd. C, 58.64; H, 3.41; N, 15.78; Found: C, 58.55; H, 3.22; N, 15.66 .

2-(4-(4-fluorophenyl)-5-methylthio-s-triazol3-ylmethyl)-3-(4-fluorophenyl)-3,4-dihydro-4oxoquinazoline (15)

To a mixture of 8 ( $1 \mathrm{mmol})$ and fused sodium acetate $(2 \mathrm{mmol})$, methyl iodide $(1 \mathrm{mmol})$ was added. The mixture was heated for 2 hours, cooled to RT and poured on crushed ice, then scratched with a glass rod and kept overnight in a refrigerator. The white solid was filtered and recrystallized from ethanol to give compound 15 in $65 \%$ yield. m.p. 185-187 ${ }^{\circ} \mathrm{C}$; IR: 1683 (C=O); ${ }^{1} \mathrm{H}-\mathrm{NMR}: 2.43$ (s, 3H), 4.28 (s, 2H), 6.92-8.23 (m, 8H, Ar-H), 8.52-8.56 (m, $4 \mathrm{H}, \mathrm{Ar}-\mathrm{H}$ of quinazoline); ${ }^{13} \mathrm{C}-\mathrm{NMR}: 14.81,21.42$, $113.42,115.51,115.52,115.72,115.73,120.82$, $125.52,126.26,126.71,127.33,128.35,128.41$, $130.11,130.14,140.11,141.42,146.91,147.33$, 153.01, 159.32, 162.91, 162.94; Mass (m/z): 493 $\left(\mathrm{M}^{+}\right)$; Elemental Analysis $\left.\left(\mathrm{C}_{24} \mathrm{H}_{17} \mathrm{~F}_{2} \mathrm{~N}_{5} \mathrm{OS}\right)_{2}\right)$ : Calcd. C, 58.41; H, 3.47; N, 14.19; Found: C, 58.22; H, 3.55; $\mathrm{N}, 14.20$.

2-(4-(4-fluorophenyl)-1-morpholinomethyl)5-thioxo- $\Delta$-s-triazolin-3-ylmethyl)-4-(4-

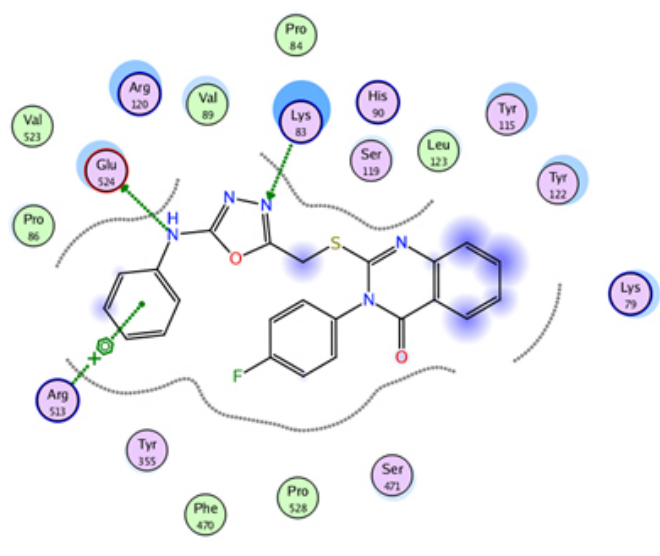

Fig. 4

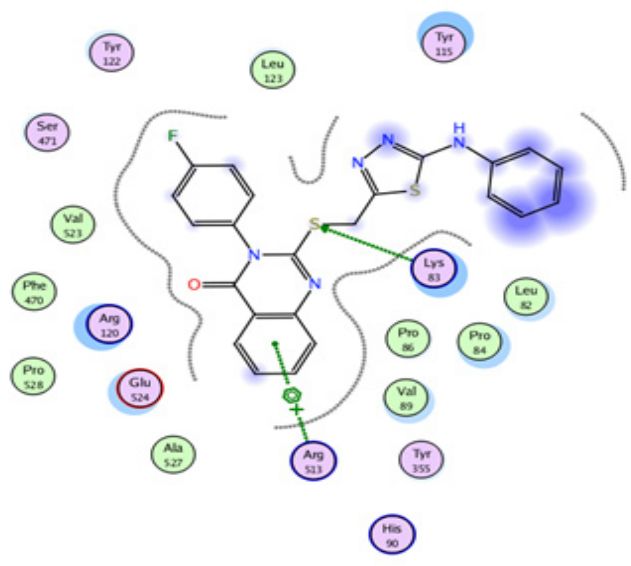

Fig. 5 
fluorophenyl)-3,4-dihydro-4-oxoquinazolin (16) An ethanolic solution of $\mathbf{8}$ was stirred with $37 \%$ formaldehyde solution $(1 \mathrm{ml})$ and ethanolic solution of morpholine ( $9 \mathrm{mmol}$ ) for 2 hours at RT. The mixture was kept in a refrigerator overnight and the precipitated solid was filtered, washed with cold ethanol and recrystallized from ethanol to give compound 16 in $90 \%$ yield. m.p. $215-217^{\circ} \mathrm{C}$; IR: 1683 $(\mathrm{C}=\mathrm{O})$; ${ }^{1} \mathrm{H}-N M R: 2.76(\mathrm{~m}, 4 \mathrm{H}), 3.76(\mathrm{~m}, 4 \mathrm{H}), 4.21$ (s, 2H), 6.92-8.23 (m, 8H, Ar-H), 8.52-8.56 (m, 4H, Ar-H of quinazoline); ${ }^{13} \mathrm{C}-\mathrm{NMR}: 2.31,2.32,2.38,2.39$, 19.21, 24.42, 36.92, 59.21, 104.32, 105.81, 113.42, $115.11,115.72,115.73,126.33,126.62,127.33$, 128.32, 128.71, 130.11, 130.13, 133.41, 141.42, 146.92, 151.01, 159.32, 160.41, 162.43; Mass (m/z): $578\left(\mathrm{M}^{+}\right)$; Elemental Analysis $\left(\mathrm{C}_{28} \mathrm{H}_{24} \mathrm{~F}_{2} \mathrm{~N}_{6} \mathrm{O}_{2} \mathrm{~S}_{2}\right)$ : Calcd. C, 58.18; H, 4.18; N, 14.52; Found: C, 58.17; $\mathrm{H}, 4.11 ; \mathrm{N}, 14.57$.

2-(4-(4-Fluorophenyl)-3-methyl-5-thioxo- $\Delta$-striazolin-3-ylmethyl)-3-(4-fluorophenyl)-3,4dihydro-4-oxoquinazoline (17)

The solution of $15(5 \mathrm{mmol})$ and methyl iodide $(9 \mathrm{mmol})$ in DMF $(20 \mathrm{ml})$ was heated for one hour. The mixture was poured into ice cold water and the precipitate was recrystallized from ethanol to give compound 17 in $60 \%$ yield. m.p. $196-198{ }^{\circ} \mathrm{C}$; IR: $1682(\mathrm{C}=\mathrm{O}), 1140$ and $1470(\mathrm{C}=\mathrm{S})$; ${ }^{1} \mathrm{H}-\mathrm{NMR}$ : 2.55 (s, 3H), 4.29 (s, 2H), 6.92-8.29 (m, 8H, Ar-H), 8.51-8.57 (m, 4H, Ar-H of quinazoline); Elemental Analysis $\left(\mathrm{C}_{24} \mathrm{H}_{17} \mathrm{~F}_{2} \mathrm{~N}_{5} \mathrm{OS}_{2}\right)$ : Calcd. $\mathrm{C}, 58.41 ; \mathrm{H}, 3.47$; N, 14.19; Found: C, 58.41; H, 3.47; N, 14.11.

\section{In Vitro Anti-inflammatory Activity}

It was carried out by the colorimetric COX (ovine) inhibitor screening assay adopting the reported procedure ${ }^{10-12}$.

\section{Docking Methodology}

Molecular modelling was performed as provided in our previous report ${ }^{8}$.

\section{RESULTS AND DISCUSSIONS}

\section{Chemistry}

Compound 1 was obtained via the reaction of anthranilic acid and 4-fluorophenyl isothiocyante (Scheme 1). The IR spectra of compound 1 revealed a signal at $1690 \mathrm{~cm}^{-1}$ corresponding to $\mathrm{C}=\mathrm{O}$ of quinazolinone 1 . Reaction of compound 1 with ethyl bromoacetate gave the corresponding ester 2 which on reaction with hydrazine hydrate in ethanol produced the corresponding hydrazide 3 . Reaction of compound 3 with 4-fluorobenzaldehyde produced the corresponding hydrazone derivative 4 . On the other hand, treatment of 1 with 2-chloro-N(substitutedphenyl)anilides gave the corresponding acetamide derivatives $5 \mathrm{a}-\mathrm{c}$. Moreover, reaction of 1 with 4-fluorophenacyl bromide gave compound 6 (Scheme 1).

Scheme 2 was achieved by reaction of compound 3 with 4-fluorophenylisothiocyanate to produce the carbothioamide derivative 7 which was used as starting compound for the synthesis of the

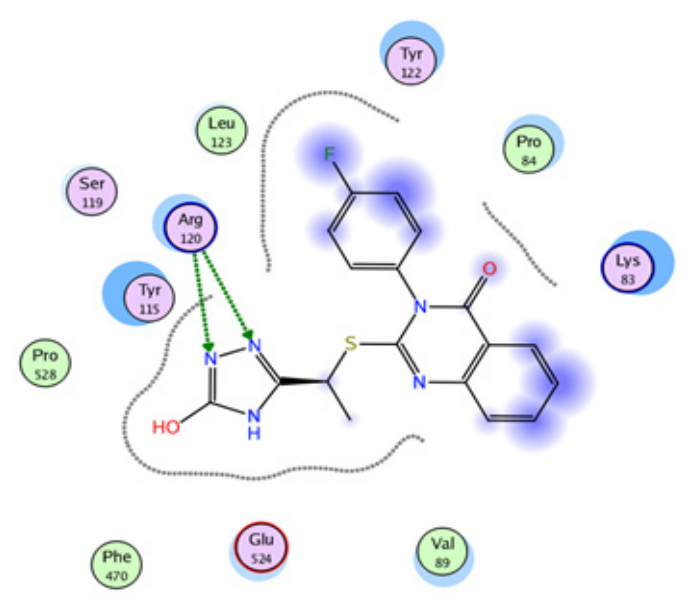

Fig.6

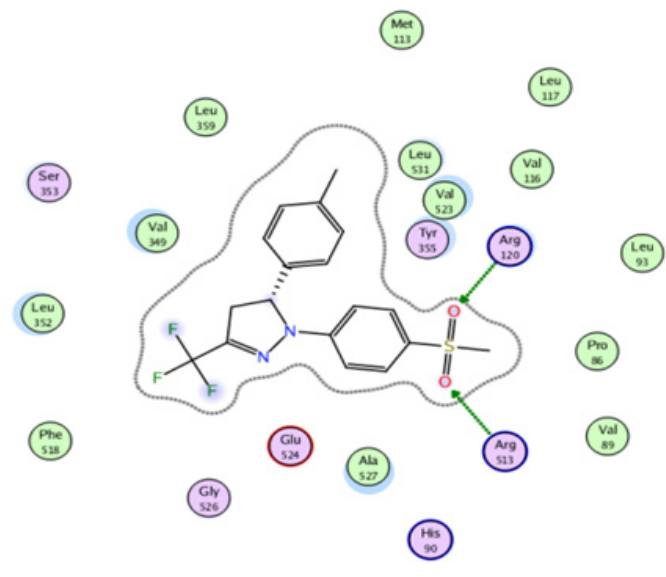

Fig. 7: Docked pose of cyclooxygenase enzyme with Celecoxib (2D). 
corresponding triazole, thiadiazole and oxadiazole derivatives. Compound 7 was reacted with aqueous $\mathrm{KOH}$ under refluxed to give the corresponding triazole derivative 8 . Similarly, when compound 7 was stirred with concentrated sulfuric acid, it gave the corresponding 1,3,4-thiadiazole derivative 9. Finally, the compound 7 was cyclodesulfurised by hrating under reflux with DCCD in toluene to give the oxadiazole derivative 10 .

The compound 11 was prepared by the reaction of the compound 1 with ethyl-2bromopropanoate (Scheme 3) to give the ester 11 which was converted to the corresponding semicarbazide 12 by the reaction with semicarbazide. The corresponding hydroxyl triazole derivative 13 was obtained by cyclocondenzation of 12 with aqueous $\mathrm{NaOH}$.

The triazole derivative 8 was subjected to cyanomethylation using acrylonitrile or aminomethylation using Mannich reaction conditions to afford the products 14 and 16 , respectively. Methylation of 8 in acetone in the presence of sodium acetate resulted in the kinetically controlled reaction product 15 as proved by the conversion of 15 to the thermodynamically controlled reaction product 16 upon heating in dimethylformamide in the presence of methyl iodide, as what have been reported by Molina and Alajarin ${ }^{13}$. Structure elucidation of the $\mathrm{N}$-substitution was confirmed by elemental analysis as well as spectral data.

\section{In vitro COX inhibition assay}

The highly scored docked derivatives 4, 8 , 9,10 , and 13 were evaluated using an in vitro COX-1 / COX-2 inhibition assay (Table 1).

\section{Molecular Modelling Studies}

These were performed using MOE 2007.09 program $^{14}$. The crystallographic enzyme ligand complex with SC-558 was obtained from the RCSB protein data bank (PDB entry $\left.\mathrm{ICX}_{2}\right)^{15,16}$.

\section{CONCLUSION}

In the present study, novel fluorinated quinazolinones having triazole, thiadiazole, and oxadiazole rings were synthesized and evaluated for their in vitro anti-inflammatory activity. Molecular modelling studies were performed to examine the selectivity on cyclooxygenase 2 enzyme. Compounds 4, 8, 9, 10, and 13 showed interesting anti-inflammatory activity and a good binding with the COX-2 enzyme. Therefore, these compounds may represent lead compounds for developing anti-inflammatory agents with high binding affinity with the receptor and no side effects. Also, fluorine will very likely continue to contribute in drug design and discovery by playing multifaceted ${ }^{17,18}$ roles in enhancing future medical advances.

\section{ACKNOWLEDGEMENTS}

The authors gratefully acknowledge the approval and support of this research study by the grant no. PHM-2016-1-6-F-5682 K.S.A. at Northern Border University, Arar from the Deanship of Scientific Research.

\section{REFERENCES}

1. Lee E.S., Kim S.I, Lee S.H., Jeong T.C., Moon T.C., Chang H.W., Jahng Y.D., Bull. Korean Chem. Soc.2005,26(12), 1975-1980.

2. Jahng Y., Arch. Pharm. Res.2013, 36(5), 517535.

3. Abdel-Aziz A.A., Abou-Zeid L.A., ElTahir K.E., Mohamed M.A., Abu El-Enin M.A., El-Azab A.S. Bioorg. Med. Chem.2016, 24(16), 3818-
3828.

4. Mahin R., Ali D. J. Fluorine Chem.2017,193, 89-97.

5. Wei W., Yuan Z., Hao P., Hong-Wu H., XingTao L. J. Fluorine Chem.2017,193, 8-16.

6. Shah P., Westwell A.D. J. Enzyme Inhib. Med. Chem.2007,22(5), 527-540.

7. Said A.E., Hamdy K.T., Mustafa T.U. J. 
Fluorine Chem.2014,161, 87-94.

8. Said A.H.E., Zakaria K.A.E., Nermine A.O., Jasmine L., Mohamed A.K., Hamdy K.T. Bioorg. Chem.2015,58, 104-116.

9. Said A.E., Hamdy K.T., Mahmoud M.E.M. Orient. J. Chem.2015, 31(2), 709-718.

10. El-Sayed M.A., Abdel-Aziz N.I., Abdel-Aziz A.A., El-Azab A.S., Asiri Y.A., Eltahir K.E. Bioorg. Med. Chem.2011, 19(11), 34163424.

11. Alanazi A.M., El-Azab A.S., Al-Suwaidan I.A., ElTahir K.E., Asiri Y.A., Abdel-Aziz N.I., Abdel-Aziz A.A. Eur. J. Med. Chem.2015,92, 115-123.

12. Uddin M.J., Rao P.N., KnausE.E..Bioorg. Med. Chem.2004,12(22), 5929-5940.

13. Molina P., Alajarin M. Synthesis1983, 5, 414-
415.

14. https://www.chemcomp.com/MOEMolecular_Operating_Environment.htm

15. Kurumbail R.G., Stevens A.M., Gierse J.K., McDonald J.J., Stegeman R.A., Pak J.Y., Gildehaus D., Miyashiro J.M., Penning T.D., Seibert K., Isakson P.C., Stallings W.C. Nature.1996, 384(6610), 644-648.

16. Xu S., Hermanson D.J., Banerjee S., Ghebreselasie K., Clayton G.M., Garavito R.M., Marnett L.J. J. Biol. Chem.2014, 289(10), 6799-6808.

17. Iwao O. J. Org. Chem.2013, 78(13), 63586383.

18. Fyaz M.D. J. Fluorine Chem.2002,118, 1-2, 27-33. 